\title{
AN EXTENSION THEOREM FOR NORMAL FUNCTIONS
}

\author{
PENTTI JÄRVI
}

(Communicated by Irwin Kra)

\begin{abstract}
Given a domain $\Omega \subset \mathbf{C}^{n}$, an analytic subvariety $V$ of $\Omega$ and a normal function $f: \Omega \backslash V \rightarrow \widehat{\mathbf{C}}$, we show that $f$ can be extended to a holomorphic mapping $f^{*}: \Omega \rightarrow \widehat{\mathbf{C}}$ provided the singularities of $V$ are normal crossings.
\end{abstract}

1. As an extension of the big Picard theorem, Lehto and Virtanen showed [5, Theorem 9] that isolated singularities are removable for normal meromorphic functions. It is the purpose of this note to give a generalization of this result for functions defined in subdomains of $\mathbf{C}^{n}$. It is conceivable that the notion of normality can be generalized in various ways to higher dimensions. Here we adopt the definition of Cima and Krantz [1, p. 305].

Let $D \subset \mathbf{C}$ be the open unit disc, and let $\Omega \subset \mathbf{C}^{n}$ be a domain. The infinitesimal form of the Kobayashi metric for $\Omega$ at $z \in \Omega$ in the direction $\xi \in \mathbf{C}^{n}$ is defined to be

$$
\begin{array}{r}
F_{\Omega}(z, \xi)=\inf \left\{|\xi| /\left|f^{\prime}(0)\right| \mid f: D \rightarrow \Omega \text { holomorphic, } f(0)=z,\right. \\
\text { and } \left.f^{\prime}(0) \text { is a positive multiple of } \xi\right\} .
\end{array}
$$

Here | $\mid$ stands for the Euclidean length. Further, let $z \in \widehat{\mathbf{C}}=\mathbf{C} \cup\{\infty\}$ and let $\eta \in \mathbf{C}$ be thought of as a tangent vector to $\widehat{\mathbf{C}}$ at $z$. Then the infinitesimal form of the spherical metric at $z$ is defined by

$$
|\eta|_{\mathrm{sph}, z}=|\eta| /\left(1+|z|^{2}\right) .
$$

Now suppose that $f$ is a holomorphic mapping of $\Omega$ into $\widehat{\mathbf{C}}$, and let $f^{\prime}$ denote the matrix $\left(\partial f / \partial z_{i}\right)$. Then $f$ is said to be normal provided there exists a constant $C$ such that

$$
\left|f^{\prime}(z) \cdot \xi\right|_{\mathrm{sph}, f(z)} \leq C \cdot F_{\Omega}(z, \xi) \quad \text { for all } z \in \Omega \text { and all } \xi \in \mathbf{C}^{n} .
$$

The minimum of those constants $C$, for which (1) holds true, is called the order of normality of $f$ and denoted by $C_{f}$.

REMARK 1. Suppose $f$ is a holomorphic mapping of $\Omega$ into $\widehat{\mathbf{C}}$ such that $\widehat{\mathbf{C}} \backslash f(\Omega)$ contains three points $a_{1}, a_{2}$ and $a_{3}$. Then $f$ is normal. First, the distancedecreasing property of the Kobayashi metric yields

$$
F_{\widehat{\mathbf{C}} \backslash\left\{a_{1}, a_{2}, a_{3}\right\}}\left(f(z), f^{\prime}(z) \cdot \xi\right) \leq F_{\Omega}(z, \xi) \quad \text { for all } z \in \Omega \text { and all } \xi \in \mathbf{C}^{n} .
$$

Received by the editors May 8, 1987 and, in revised form, July 2, 1987.

1980 Mathematics Subject Classification (1985 Revision). Primary 32H25; Secondary 32H20.

Key words and phrases. Normal function, Kobayashi metric.

(C) 1988 American Mathematical Society $0002-9939 / 88 \$ 1.00+\$ .25$ per page 
Further, making use of the homogeneity and the continuity of $F_{\widehat{\mathbf{C}} \backslash\left\{a_{1}, a_{2}, a_{3}\right\}}$ and the spherical metric as well as the fact that

$$
|1|_{\mathrm{sph}, z} / F_{\widehat{\mathbf{C}} \backslash\left\{a_{1}, a_{2}, a_{3}\right\}}(z, 1) \rightarrow 0 \quad \text { as } z \rightarrow a_{j}, j=1,2,3,
$$

one readily finds a constant $C$ such that

(3) $|\eta|_{\mathrm{sph}, z} \leq C \cdot F_{\widehat{\mathbf{C}} \backslash\left\{a_{1}, a_{2}, a_{3}\right\}}(z, \eta)$ for all $z \in \widehat{\mathbf{C}} \backslash\left\{a_{1}, a_{2}, a_{3}\right\}$ and all $\eta \in \mathbf{C}$.

Combining (2) and (3) gives the assertion. Another deduction of this result is given in $[\mathbf{1}$, p. 308].

2. Set $D^{*}=D \backslash\{0\}$, and let $f: D^{*} \rightarrow \widehat{\mathbf{C}}$ be normal. As noted before, $f$ extends to a function $f^{*}$ meromorphic in $D\left[5\right.$, p. 62]. Since $F_{D}(z, \eta)$ and $F_{D^{*}}(z, \eta)$ are comparable near $\partial D, f^{*}$ is normal in $D$. Moreover, the order of normality of $f^{*}$ does not deviate too much from that of $f$. More precisely, we have

LEMMA 1. Given a positive number $K$, there is a positive number $K^{\prime}$ such that any function $f$ normal in $D^{*}$ with $C_{f} \leq K$ extends to a function $f^{*}$ normal in $D$ with $C_{f^{*}} \leq K^{\prime}$.

PROOF. We begin with a quick proof of the Lehto-Virtanen extension theorem. Let $f$ be normal in $D^{*}$. Recall that

$$
F_{D^{*}}(z, \xi)=\text { hyperbolic metric of } D^{*}=\frac{|\xi|}{|z| \log (1 /|z|)} .
$$

Hence the hyperbolic area of $D^{*}(r)=\{z \in \mathbf{C}|0<| z \mid<r\}$ is finite for every $r<1$. By (1), we have

$$
\iint_{D^{*}(r)} \frac{\left|f^{\prime}(z)\right|^{2}}{\left(1+|f(z)|^{2}\right)^{2}} d x d y<\infty
$$

By the big Picard theorem, the singularity at 0 is inessential.

Fix $K>0$, and let $f: D^{*} \rightarrow \widehat{\mathbf{C}}$ be normal with $C_{f} \leq K$. Again, let $f^{*}$ stand for the extended function. Since the spherical metric is invariant under the rotations of the sphere, we may assume that $f^{*}(0)=0$. Clearly, it is sufficient to exhibit an $R, 0<R<1$, depending only on $K$, such that $\left|f^{*}(z)\right|<1$ for $z \in D(R)=\{z \in \mathbf{C}|| z \mid<R\}$. By assumption,

$$
\frac{\left|f^{\prime}(z)\right|}{1+|f(z)|^{2}} \leq K \cdot \frac{1}{|z| \log (1 /|z|)} \quad \text { for all } z \in D^{*} \text {. }
$$

Set $\gamma_{r}=\{z \in \mathbf{C}|| z \mid=r\}, 0<r<1$, and let $s\left(f^{*}\left(\gamma_{r}\right)\right)$ denote the spherical length of $f^{*}\left(\gamma_{r}\right)$. We claim that $R=e^{-8 K}$ does the job. Suppose, on the contrary, that $\left|f^{*}(z)\right| \geq 1$ for some $z \in D(R)$. Pick out $z_{0} \in D(R)$ such that $\left|f^{*}\left(z_{0}\right)\right|=1$ and $\left|f^{*}(z)\right|<1$ for $|z|<\left|z_{0}\right|$. A simple estimate based on (4) gives $s\left(f^{*}\left(\gamma_{\left|z_{0}\right|}\right)\right)<\pi / 4$. Since the spherical distance of 0 and $f\left(z_{0}\right)$ is $\pi / 2, f^{*}\left(\gamma_{\left|z_{0}\right|}\right)$ lies in the half plane $\operatorname{Re} \overline{f\left(z_{0}\right)} z>0$. Therefore, the winding number of $f^{*}\left(\gamma_{\left|z_{0}\right|}\right)$ with respect to 0 (in $\mathbf{C}$ ) is 0 . This contradiction with $f^{*}(0)=0$ completes the proof.

The next lemma is readily deduced by elementary considerations on the Kobayashi metric. We omit the proof. 
LEMMA 2. Let $f:\left(D^{*}\right)^{n} \rightarrow \widehat{\mathbf{C}}$ be normal.

(1) For every $k \in\{1, \ldots, n\}$ and every $\left(a_{1}, \ldots, a_{n-1}\right) \in\left(D^{*}\right)^{n-1}$, the map $z \mapsto f\left(a_{1}, \ldots, a_{k-1}, z, a_{k}, \ldots, a_{n-1}\right), D^{*} \rightarrow \widehat{\mathbf{C}}$, is a normal function.

(2) For every $k \in\{1, \ldots, n\}$ and every $a \in D^{*}$ the map $\left(z_{1}, \ldots, z_{n-1}\right) \mapsto$ $f\left(z_{1}, \ldots, z_{k-1}, a, z_{k}, \ldots, z_{n-1}\right),\left(D^{*}\right)^{n-1} \rightarrow \widehat{\mathbf{C}}$, is a normal function.

(3) For every $a=\left(a_{1}, \ldots, a_{n}\right) \in \partial D^{n}$ with $a_{i} \neq 0, i=1, \ldots, n$, the map $z \mapsto f\left(a_{1} z, \ldots, a_{n} z\right), D^{*} \rightarrow \widehat{\mathbf{C}}$, is a normal function.

Further, the orders of normality of all these functions are bounded above by that of $f$.

3. Let $\Omega \in \mathbf{C}^{n}$ be a domain and let $V \subset \Omega$ be an analytic subvariety of codimension one. The singularities of $V$ are said to be normal crossings provided $\Omega \backslash V$ is locally biholomorphic to $\left(D^{*}\right)^{k} \times D^{n-k}$ for some $k \in\{0, \ldots, n\}$. Our main theorem reads as follows.

THEOREM 1. Let $\Omega \subset \mathbf{C}^{n}$ be a domain and let $V \subset \Omega$ be an analytic subvariety of codimension one, whose singularities are normal crossings. Suppose $f: \Omega \backslash V \rightarrow$ $\widehat{\mathbf{C}}$ is normal. Then $f$ extends to a holomorphic mapping $f^{*}: \Omega \rightarrow \widehat{\mathbf{C}}$.

PrOOF. Since the problem is of a local nature and the inclusion mapping is distance-decreasing (in the Kobayashi metrics), we may assume that $\Omega=D^{n}$ and $D^{n} \backslash V=\left(D^{*}\right)^{n}$.

The proof will be by induction on $n$. The case $n=1$ is part of Lemma 1. So let $n \geq 2$ and assume the extension is possible for $1, \ldots, n-1$. Let $a=\left(a_{1}, \ldots, a_{n}\right) \in V \backslash\{0\}$, and choose $k \in\{1, \ldots, n\}$ such that $a_{k} \neq 0$. Consider the mapping $f_{a_{k}}:\left(z_{1}, \ldots, z_{n-1}\right) \mapsto f\left(z_{1}, \ldots, z_{k-1}, a_{k}, z_{k}, \ldots, z_{n-1}\right),\left(D^{*}\right)^{n-1} \rightarrow \widehat{\mathbf{C}}$. It follows from Lemma 2 that $f_{a_{k}}$ is normal. Hence, by the induction hypothesis, $f_{a_{k}}$ admits a holomorphic extension $f_{a_{k}}^{*}: D^{n-1} \rightarrow \widehat{\mathbf{C}}$. We set $f^{*}(a)=$ $f_{a_{k}}^{*}\left(a_{1}, \ldots, a_{k-1}, a_{k+1}, \ldots, a_{n}\right)$. We will show that the extended mapping is holomorphic on $D^{n} \backslash\{0\}$. By the Riemann extension theorem, it suffices to prove that $f^{*}$ is continuous, i.e., $\mathrm{Cl}(f ; a)$, the cluster set of $f$ at any $a \in V \backslash\{0\}$ reduces to a singleton (of course, this also shows that the extension does not depend on the choice of $k$ ).

So let $a, k$ and $f^{*}(a)$ be as above. Pick $\varepsilon>0$. Set

$$
\chi(z, w)=\frac{|z-w|}{\left(1+|z|^{2}\right)^{1 / 2}\left(1+|w|^{2}\right)^{1 / 2}} \quad \text { for } z, w \in \widehat{\mathbf{C}}
$$

and $B(a, \delta)=\left\{z \in \mathbf{C}^{n}|| z-a \mid<\delta\right\}$. By Lemma 2, the family

$$
\left\{z \mapsto f\left(b_{1}, \ldots, b_{k-1}, z, b_{k}, \ldots, b_{n-1}\right) \mid\left(b_{1}, \ldots, b_{n-1}\right) \in\left(D^{*}\right)^{n-1}\right\}
$$

is equicontinuous at $a_{k}$. Hence there exists a positive $\delta$ such that

$$
\chi\left(f(z), f\left(z_{1}, \ldots, z_{k-1}, a_{k}, z_{k+1}, \ldots, z_{n}\right)\right)<\varepsilon / 2
$$

and

$$
\chi\left(f\left(z_{1}, \ldots, z_{k-1}, a_{k}, z_{k+1}, \ldots, z_{n}\right), f^{*}(a)\right)<\varepsilon / 2 \text { for } z=\left(z_{1}, \ldots, z_{n}\right) \in B(a, \delta) \backslash V .
$$

Thus $\chi\left(f(z), f^{*}(a)\right)<\varepsilon$ for $z \in B(a, \delta) \backslash V$. It follows that $\mathrm{Cl}(f ; a)=f^{*}(a)$.

It remains to extend $f$ to 0 . First, we infer from Lemmas 2 and 1 that $f(z, \ldots, z)$ tends to a limit, say $w_{0}$, as $z \rightarrow 0$. It suffices to show that $\mathrm{Cl}(f ; 0)=w_{0}$. Let $\varepsilon>0$. 
Consider the mappings described in Lemma 2 (3), or rather their counterparts for the restrictions of $f$ to the hyperplanes of the form $\left\{z_{n}=a\right\}, a \in D^{*}$. By Lemma 1, all of them extend holomorphically to 0 . Moreover, it follows from Lemmas 2 and 1 that the extensions constitute an equicontinuous family at 0 $(\in \mathbf{C})$. Therefore, we find a positive $\delta$ such that $\chi\left(f(z), f^{*}\left(0, \ldots, 0, z_{n}\right)\right)<\varepsilon / 3$, $\chi\left(f^{*}\left(0, \ldots, 0, z_{n}\right), f\left(z_{n}, \ldots, z_{n}\right)\right)<\varepsilon / 3$ and $\chi\left(f\left(z_{n}, \ldots, z_{n}\right), w_{0}\right)<\varepsilon / 3$ for $z=$ $\left(z_{1}, \ldots, z_{n}\right) \in B(0, \delta) \backslash V$. Therefore $\chi\left(f(z), w_{0}\right)<\varepsilon$ for $z \in B(0, \delta) \backslash V$. Hence $\mathrm{Cl}(f ; 0)=w_{0}$. This completes the proof.

REMARK 2. Set $V=\left\{\left(z_{1}, z_{2}\right) \in \mathbf{C}^{2} \mid z_{1} z_{2}\left(z_{1}-z_{2}\right)=0\right\}$ and consider the mapping $f: D^{2} \backslash V \rightarrow \widehat{\mathbf{C}},\left(z_{1}, z_{2}\right) \mapsto z_{1} / z_{2}$. Since $f$ omits the values 0,1 and $\infty$ in $D^{2} \backslash V$, it is normal by Remark 1 . Yet $f$ does not extend to a holomorphic map $D^{2} \rightarrow \widehat{\mathbf{C}}$. Accordingly, we cannot dispense with some restrictions on the singularities of $V$ in Theorem 1.

REMARK 3. One may ask whether the extended function is normal in $\Omega$ (provided $\Omega$ is hyperbolic). However, it seems that this need not be the case even in dimension one.

REMARK 4. Results related to Theorem 1 can be found in [2, 3 and 4]. Cf. in particular [2, Theorem 2].

In the counterexample discussed above the function involved is the restriction to $D^{2} \backslash V$ of a meromorphic function, i.e., a function with "indeterminacies". This is always the case as shown by

THEOREM 2. Let $\Omega \subset \mathbf{C}^{n}$ be a domain and let $V$ be a subvariety of $\Omega$. Suppose $f: \Omega \backslash V \rightarrow \widehat{\mathbf{C}}$ is normal. Then $f$ extends to a meromorphic function in $\Omega$.

PROOF. Denote by $S(V)$ the set of singular points of $V$. By Theorem $1 f$ extends to a holomorphic mapping of $\Omega \backslash S(V)$ into $\widehat{\mathbf{C}}$. Thus $f$ can be regarded as a meromorphic function in $\Omega \backslash S(V)$. Since $\operatorname{dim} S(V) \leq n-2$ [6, p. 144], $f$ extends to a function meromorphic in $\Omega$ [6, p. 149].

\section{REFERENCES}

1. J. A. Cima and S. G. Krantz, The Lindelöf principle and normal functions of several complex variables, Duke Math. J. 50 (1983), 303-328.

2. P. Kiernan, Extensions of holomorphic maps, Trans. Amer. Math. Soc. 172 (1972), 347-355.

3. S. Kobayashi, Hyperbolic manifolds and holomorphic mappings, Pure Appl. Math., no. 2, Dekker, New York, 1970.

4. M. H. Kwack, Generalization of the big Picard theorem, Ann. of Math. (2) 90 (1969), 9-22.

5. O. Lehto and K. I. Virtanen, Boundary behaviour and normal meromorphic functions, Acta Math. 97 (1957), 47-65.

6. W. Rothstein und K. Kopfermann, Funktionentheorie mehrerer komplexer Veränderlicher, Bibliographisches Institut, Mannheim, 1982.

Department of Mathematics, University of Helsinki, SF-00100 Helsinki 10, FINLAND 